\title{
AN EXPLICIT DESCRIPTION OF THE SIMPLICIAL GROUP $K(A, n)$
}

\author{
MIHAI D. STAIC \\ (Received 6 September 2011; accepted 1 March 2013; first published online 7 June 2013) \\ Communicated by C. Hodgson
}

\begin{abstract}
We give an explicit construction for a $K(A, n)$ simplicial group and explain its topological interpretation.

2010 Mathematics subject classification: primary 18G30; secondary $20 \mathrm{~J} 06$.

Keywords and phrases: simplicial groups, group cohomology, Hopf algebras.
\end{abstract}

\section{Introduction}

Simplicial groups are purely algebraic objects that are used in algebraic topology to formulate classification results. Just like for topological spaces one can talk about the $n$th homotopy group of a simplicial group. A $K(A, n)$ simplicial group is determined by the fact that $\pi_{i}(K(A, n))=0$ if $i \neq n$ and $\pi_{n}(K(A, n))=A$. In other words, it is the algebraic object corresponding to an Eilenberg-MacLane space $K(A, n)$. If $A$ is a fixed commutative group, there is an iterating procedure that gives a presentation of the simplicial group of $K(A, n)$ (see [7]). Unfortunately, some of the topological nature of simplicial objects is lost in the iterating process. There are also explicit descriptions of $K(A, n)$ (see, for example, [4] or [9]), but again the topological flavor is not transparent.

In this paper we give a new explicit description of the simplicial group $K(A, n)$. The main advantage of our presentation is that it has a nice topological interpretation. Also the description is very simple and is presented in terms of the generating maps of the simplicial category $\Delta$.

In the first section we recall basic definitions, properties and examples of simplicial groups. The second section starts with the description of $K(A, 2)$. This construction appears in a nonexplicit way in [8] and it was the starting point for this paper. We show that $K(A, 2)$ is a cyclic object. For a better understanding of our general construction

Research partially supported by the CNCSIS project 'Hopf algebras, cyclic homology and monoidal categories' contract nr. 560/2009, CNCSIS code ID_69.

(C) 2013 Australian Mathematical Publishing Association Inc. 1446-7887/2013 \$16.00 
we also present the case of $K(A, 3)$. In the third section we give the description of the simplicial group $K(A, n)$. The punch line is in the way we chose the index for the elements of the group $K(A, n)_{q}=A^{\left(\begin{array}{c}q \\ n\end{array}\right)}$. We explain the topological interpretation and discuss some possible applications.

The last section deals with a similar construction in the context of Hopf algebras. More precisely, to every commutative Hopf algebra $H$ we associate a cyclic object ${ }_{2} K(H)$. If $H$ is the group algebra $k[A]$ associated to a commutative group $A$, then the cyclic object ${ }_{2} K(H)$ is just the linearization of the cyclic object $K(A, 2)$ mentioned above.

\section{Preliminaries}

We recall from $[6,7]$ a few facts about simplicial groups. First, we need the definition of the simplicial category $\Delta$. The objects in $\Delta$ are the finite ordered sets $\bar{n}=\{0,1, \ldots, n\}$. The morphisms are the order preserving maps. One can show that any morphism in $\Delta$ can be written as a composition of maps $d^{i}: \bar{n} \rightarrow \overline{n+1}$ and $s^{i}: \bar{n} \rightarrow \overline{n-1}$, where

$$
\begin{aligned}
& d^{i}(u)= \begin{cases}u & \text { if } u<i, \\
u+1 & \text { if } u \geq i\end{cases} \\
& s^{i}(u)= \begin{cases}u & \text { if } u \leq i, \\
u-1 & \text { if } u>i\end{cases}
\end{aligned}
$$

A simplicial group is a functor $K: \Delta^{\mathrm{op}} \rightarrow G r$. More explicitly, a simplicial group is a set of groups $X_{q}, q \geq 0$, together with a collection of group morphisms $\partial_{i}: X_{q} \rightarrow X_{q-1}$ and $s_{i}: X_{q} \rightarrow X_{q+1}$ for all $0 \leq i \leq q$ such that the following identities hold:

$$
\begin{aligned}
& \partial_{i} \partial_{j}=\partial_{j-1} \partial_{i} \quad \text { if } i<j, \\
& s_{i} s_{j}=s_{j+1} s_{i} \quad \text { if } i \leq j, \\
& \partial_{i} s_{j}=s_{j-1} \partial_{i} \quad \text { if } i<j, \\
& \partial_{j} s_{j}=\partial_{j+1} s_{j}=\text { id, } \\
& \partial_{i} s_{j}=s_{j} \partial_{i-1} \quad \text { if } i>j+1 .
\end{aligned}
$$

Let $\mathbf{K}=\left(K_{q}, \partial_{i}, s_{i}\right)$ be a simplicial group. We denote

$$
\bar{K}_{q}=K_{q} \cap \operatorname{Ker}\left(\partial_{0}\right) \cap \cdots \cap \operatorname{Ker}\left(\partial_{q-1}\right)
$$

One can show that $\partial_{q+1}\left(\bar{K}_{q+1}\right) \subseteq \bar{K}_{q}$ and so $\overline{\mathbf{K}}=\left(\bar{K}_{q+1}, \partial_{q+1}\right)$ is a chain complex. The homotopy groups of the simplicial group $\mathbf{K}$ are defined by

$$
\pi_{q}(\mathbf{K})=H_{q}(\overline{\mathbf{K}})
$$

If $q \geq 2$ then $\pi_{q}(\mathbf{K})$ is an abelian group. If $\mathbf{K}$ has the property that $\pi_{i}(\mathbf{K})=0$ if $i \neq n$ and $\pi_{n}(\mathbf{K})=G$ then it is called an Eilenberg-MacLane simplicial group and is denoted by $K(G, n)$. 
We recall from [7] the construction of the $K(G, 1)$ simplicial group. Define

$$
K_{q}=G^{q},
$$

where the elements of $G^{q}$ are $q$-tuples $\left(g_{0}, g_{1}, \ldots, g_{q-1}\right)$. For every $0 \leq i \leq q-1$ define

$$
\begin{gathered}
\partial_{i}: G^{q} \rightarrow G^{q-1} \quad \text { and } \quad s_{i}: G^{q} \rightarrow G^{q+1}, \\
\partial_{0}\left(g_{0}, g_{1}, \ldots, g_{q-1}\right)=\left(g_{1}, \ldots, g_{q-1}\right), \\
\partial_{i}\left(g_{0}, g_{1}, \ldots, g_{q-1}\right)=\left(g_{0}, \ldots, g_{i-1} g_{i}, \ldots, g_{q-1}\right), \\
\partial_{q}\left(g_{0}, g_{1}, \ldots, g_{q-1}\right)=\left(g_{0}, \ldots, g_{q-2}\right)
\end{gathered}
$$

and

$$
\begin{aligned}
s_{0}\left(g_{0}, g_{1}, \ldots, g_{q-1}\right) & =\left(1, g_{0}, \ldots, g_{q-1}\right), \\
s_{i}\left(g_{0}, g_{1}, \ldots, g_{q-1}\right) & =\left(g_{0}, \ldots, g_{i-1}, 1, g_{i}, \ldots, g_{q-1}\right), \\
s_{q}\left(g_{0}, g_{1}, \ldots, g_{q-1}\right) & =\left(g_{0}, \ldots, g_{q-1}, 1\right) .
\end{aligned}
$$

One can show that if $G$ is a commutative group then $\left(K_{q}, \partial_{i}, s_{i}\right)$ is a $K(G, 1)$ simplicial group. The group structure on $K_{q}=G^{q}$ is given by the direct product. Moreover, if

$$
\begin{gathered}
\tau_{q}: K_{q}=G^{q} \rightarrow K_{q}=G^{q}, \\
\tau_{q}\left(g_{0}, g_{1}, \ldots, g_{q-1}\right)=\left(\left(g_{0} g_{1} \ldots g_{q-1}\right)^{-1}, g_{0}, \ldots, g_{q-2}\right),
\end{gathered}
$$

then $\left(K_{q}, \partial_{i}, s_{i}, \tau_{q}\right)$ is a cyclic simplicial group.

Let $(H, \Delta, \varepsilon, S)$ be a Hopf algebra. We use Sweedler's sigma notation $\Delta(h)=$ $\sum h^{\langle 1\rangle} \otimes h^{\langle 2\rangle}$. A pair $(\delta, \sigma)$ is a modular pair in involution if $\sigma \in H$ is grouplike element, $\delta: H \rightarrow k$ is a character for $H, \delta(\sigma)=1$ and $\tilde{S}_{\sigma}^{2}=$ id, where $\tilde{S}_{\sigma}(h)=$ $\sigma \sum \delta\left(h^{\langle 2\rangle}\right) S\left(h^{\langle 1\rangle}\right)$. It was proved in [5] that to a Hopf algebra $H$ and a modular pair in involution $(\delta, \sigma)$ one can associate a cyclic module $H_{n}^{(\delta, \sigma)}$. The simplicial structure is $H_{n}^{(\delta, \sigma)}=H^{\otimes n}$ and

$$
\begin{aligned}
\partial_{0}\left(h_{1} \otimes h_{2} \otimes \cdots \otimes h_{n}\right) & =\varepsilon\left(h_{1}\right) h_{2} \otimes \cdots \otimes h_{n}, \\
\partial_{i}\left(h_{1} \otimes h_{2} \otimes \cdots \otimes h_{n}\right) & =h_{1} \otimes \cdots \otimes h_{i} h_{i+1} \otimes \cdots \otimes h_{n}, \\
\partial_{n}\left(h_{1} \otimes h_{2} \otimes \cdots \otimes h_{n}\right) & =\delta\left(h_{n}\right) h_{1} \otimes \cdots \otimes h_{n-1}, \\
s_{0}\left(h_{1} \otimes h_{2} \otimes \cdots \otimes h_{n}\right) & =1 \otimes h_{1} \otimes \cdots \otimes h_{n}, \\
s_{i}\left(h_{1} \otimes h_{2} \otimes \cdots \otimes h_{n}\right) & =h_{1} \otimes \cdots h_{i} \otimes 1 \otimes h_{i+1} \otimes \cdots \otimes h_{n}, \\
s_{n}\left(h_{1} \otimes h_{2} \otimes \cdots \otimes h_{n}\right) & =h_{1} \otimes \cdots \otimes h_{n} \otimes 1 .
\end{aligned}
$$

For the cyclic action define

$$
\tau_{n}\left(h_{1} \otimes h_{2} \otimes \cdots \otimes h_{n}\right)=\sum \delta\left(h_{n}^{\langle 2\rangle}\right) S_{\sigma}\left(h_{1}^{\langle 1\rangle} h_{2}^{\langle 1\rangle} \cdots h_{n}^{\langle 1\rangle}\right) \otimes h_{1}^{\langle 2\rangle} \otimes \cdots \otimes h_{n-1}^{\langle 2\rangle} .
$$

ReMARK 2.1. When we specialize to the case $H=k[G]$, the group algebra associated to a group $G, \sigma=1$ and $\delta=\varepsilon$, we get the linearization of the cyclic simplicial group $K(G, 1)$ described above. 
Remark 2.2. If $H$ is a cocomutative Hopf algebra then $(\varepsilon, 1)$ is a modular pair in involution. The simplicial object $H^{(\varepsilon, 1)}$ has a natural structure of a symmetric object (see [6] for the definition of symmetric objects). The action of the symmetric group $\Sigma_{n+1}$ is given by

$$
\begin{gathered}
(1,2)\left(h_{1} \otimes \cdots \otimes h_{n}\right)=\sum S\left(h_{1}^{\langle 1\rangle}\right) \otimes h_{1}^{\langle 2\rangle} h_{2} \otimes \cdots \otimes h_{n}, \\
(i, i+1)\left(h_{1} \otimes \cdots \otimes h_{n}\right)=\sum h_{1} \otimes \cdots \otimes h_{i-1} h_{i}^{\langle 1\rangle} \otimes S\left(h_{i}^{\langle 2\rangle}\right) \otimes h_{i}^{\langle 3\rangle} h_{i+1} \otimes \cdots \otimes h_{n}, \\
(n, n+1)\left(h_{1} \otimes \cdots \otimes h_{n}\right)=\sum h_{1} \otimes \cdots \otimes h_{n-1} h_{n}^{\langle 1\rangle} \otimes S\left(h_{n}^{\langle 2\rangle}\right) .
\end{gathered}
$$

One can notice that the map $\tau_{n}$ is given by the action of the cycle $(1,2, \ldots, n+1) \in$ $\Sigma_{n+1}$ on $H^{\otimes n}$. And so the cyclic structure on $H^{(\varepsilon, 1)}$ is induced by the above symmetric structure.

\section{Eilenberg-MacLane simplicial groups (case $n=2$ and $n=3$ )}

3.1. $K(A, 2)$. In this section, $A$ is a commutative group. Inspired by the construction of the first $k$-invariant [3], in [8] we introduced the secondary cohomology ${ }_{2} H^{n}(A, B)$ (where $B$ is also a commutative group). We proved that to a topological space $X$ with $\pi_{1}(X)=1$ one can associate an invariant ${ }_{2} \kappa^{4} \in{ }_{2} H^{4}\left(\pi_{2}(X), \pi_{3}(X)\right)$. As a byproduct of that construction, one gets an explicit description of the simplicial group $K(A, 2)$. The basic idea is that in order to color the two-dimensional skeleton of the q-simplex $\Delta_{q}$ with elements of the group $A$, it is enough to say what the colors are for all 2-simplices of the form $[u, v, v+1]$ where $0 \leq u<v \leq q-1$. For the rest of the 2-faces, the color is determined by 'homotopy'. Take for example $\Delta_{3}$ : once we fix the color for the 2 -faces $[0,1,2],[0,2,3]$ and $[1,2,3]$ as $a_{0,1}, a_{0,2}$ and $a_{1,2}$, respectively, then the face $[0,1,3]$ must have the color $a_{0,1} a_{0,2} a_{1,2}^{-1}$. To see this we should think that $A$ is the second homotopy group $\pi_{2}(X)$ of a simply connected topological space $X$. We send the 1-skeleton of $\Delta_{3}$ to the basepoint of $X$, and three of the 2-faces of $\Delta_{3}$ according to the above prescription. If we want to have a map from $\Delta_{3}$ to $X$ we are forced to send the face $[0,1,3]$ to the element $a_{0,1} a_{0,2} a_{1,2}^{-1}$. For the case $q=4$, see [8], where these ideas were used to define the secondary cohomology of a group $A$. In general, the partial labeling of the 2-faces $[u, v, v+1]$ of $\Delta_{q}$ can be extended uniquely to a labeling of all of the 2-faces by inductively labeling the remaining faces in such a fashion that the product of all 2-faces around a 3 -simplex is $1 \in A$.

Define

$$
K_{q}=A^{q(q-1) / 2} .
$$

The elements of $A^{q(q-1) / 2}$ are $(q(q-1) / 2)$-tuples $\left(a_{u, v}\right)_{(0 \leq u<v \leq q-1)}$ with the index in the lexicographic order

$$
\left(a_{0,1}, a_{0,2}, \ldots, a_{0, q-1}, a_{1,2}, a_{1,3}, \ldots, a_{1, q-1}, \ldots, a_{q-2, q-1}\right) .
$$

For every $0 \leq i \leq q$ we define

$$
\partial_{i}: K_{q}=A^{q(q-1) / 2} \rightarrow K_{q-1}=A^{(q-1)(q-2) / 2},
$$


with $\partial_{i}\left(\left(a_{u, v}\right)_{(0 \leq u<v \leq q-1)}\right)=\left(b_{u, v}\right)_{(0 \leq u<v \leq q-2)}$, where

$$
b_{u, v}= \begin{cases}a_{u, v} & \text { if } 0 \leq u<v<i-1, \\ a_{u, v} a_{u, i} a_{v, i}^{-1} & \text { if } 0 \leq u<v=i-1, \\ a_{u, v+1} & \text { if } 0 \leq u \leq i-1<v \\ a_{u+1, v+1} & \text { if } i-1<u<v\end{cases}
$$

and

$$
s_{i}: K_{q}=A^{q(q-1) / 2} \rightarrow K_{q+1}=A^{(q+1) q / 2},
$$

with $s_{i}\left(\left(a_{u, v}\right)_{(0 \leq u<v \leq q-1)}\right)=\left(c_{u, v}\right)_{(0 \leq u<v \leq q)}$, where

$$
c_{u, v}= \begin{cases}a_{u, v} & \text { if } 0 \leq u<v<i, \\ 1 & \text { if } 0 \leq u<v=i, \\ a_{u, v-1} & \text { if } 0 \leq u<i<v, \\ a_{u, v-1} & \text { if } 0 \leq u=i<v-1, \\ 1 & \text { if } 0 \leq u=i=v-1, \\ a_{u-1, v-1} & \text { if } 0 \leq i<u<v .\end{cases}
$$

A long but straightforward computation shows that $\left(K_{q}, \partial_{i}, s_{i}\right)$ is a simplicial group. Moreover, one can see that

$$
\bar{K}_{q}=K_{q} \cap \operatorname{Ker}\left(\partial_{0}\right) \cap \cdots \cap \operatorname{Ker}\left(\partial_{q-1}\right)= \begin{cases}1 & \text { if } q \neq 2 \\ A & \text { if } q=2\end{cases}
$$

We have the following theorem.

Theorem 3.1. $\left(K_{q}, \partial_{i}, s_{i}\right)$ is a $K(A, 2)$ simplicial group.

Corollary 3.2. $\left(K_{q}, \partial_{i}, s_{i}, \tau_{q}\right)$ is a cyclic simplicial group, where $\tau_{q}: K_{q} \rightarrow K_{q}$,

$$
\begin{gathered}
\tau_{q}\left(\left(a_{u, v}\right)_{(0 \leq u<v \leq q-1)}\right)=\left(e_{u, v}\right)_{(0 \leq u<v \leq q-1)}, \\
e_{u, v}= \begin{cases}a_{v-1, v} a_{v-1, v+1} \cdots a_{v-1, q-1} a_{v, v+1}^{-1} a_{v, v+2}^{-1} \cdots a_{v, q-1}^{-1} & \text { if } 0=u<v, \\
a_{u-1, v-1} & \text { if } 0<u<v .\end{cases}
\end{gathered}
$$

3.2. $K(\boldsymbol{A}, 3)$. Just like above one can see that in order to color the 3 -skeleton of the q-simplex $\Delta_{q}$, it is enough to color all 3-simplices of the form $[u, v, w, w+1]$, where $0 \leq u<v<w \leq q-1$. The color for the other 3-faces is determined by 'homotopy'.

We define

$$
K_{q}=A^{q(q-1)(q-2) / 6} .
$$

The elements of $A^{q(q-1)(q-2) / 6}$ are $(q(q-1)(q-2) / 6)$-tuples $\left(a_{u, v, w}\right)_{(0 \leq u<v<w \leq q-1)}$ with the index in the lexicographic order

$$
\left(a_{0,1,2}, a_{0,1,3}, \ldots, a_{0,1, q-1}, a_{0,2,3}, a_{0,2,4}, \ldots, a_{q-3, q-2, q-1}\right) .
$$

For every $0 \leq i \leq q$ we put

$$
\partial_{i}: K_{q}=A^{q(q-1)(q-2) / 6} \rightarrow K_{q-1}=A^{(q-1)(q-2)(q-3) / 6},
$$


with $\partial_{i}\left(\left(a_{u, v, w}\right)_{(0 \leq u<v<w \leq q-1)}=\left(b_{u, v, w}\right)_{(0 \leq u<v<w \leq q-2)}\right.$, where

$$
b_{u, v, w}= \begin{cases}a_{u, v, w} & \text { if } 0 \leq u<v<w<i-1, \\ a_{u, v, w} a_{u, v, i} a_{u, w, i}^{-1} a_{v, w, i} & \text { if } 0 \leq u<v<w=i-1, \\ a_{u, v, w+1} & \text { if } 0 \leq u<v \leq i-1<w \\ a_{u, v+1, w+1} & \text { if } 0 \leq u \leq i-1<v<w \\ a_{u+1, v+1, w+1} & \text { if } i-1<u<v<w\end{cases}
$$

and

$$
s_{i}: K_{q}=A^{q(q-1)(q-2) / 6} \rightarrow K_{q+1}=A^{(q+1) q(q-1) / 6},
$$

with $s_{i}\left(\left(a_{u, v, w}\right)_{(0 \leq u<v<w \leq q-1)}\right)=\left(c_{u, v, w}\right)_{(0 \leq u<v<w \leq q)}$, where

$$
c_{u, v, w}= \begin{cases}a_{u, v, w} & \text { if } 0 \leq u<v<w<i, \\ 1 & \text { if } 0 \leq u<v<w=i, \\ a_{u, v, w-1} & \text { if } 0 \leq u<v<i<w, \\ a_{u, v, w-1} & \text { if } 0 \leq u<v=i<w-1, \\ 1 & \text { if } 0 \leq u<v=i=w-1 \\ a_{u, v-1, w-1} & \text { if } 0 \leq u<i<v<w, \\ a_{u, v-1, w-1} & \text { if } 0 \leq u=i<v-1<w-1, \\ 1 & \text { if } 0 \leq u=i=v-1<w-1, \\ a_{u-1, v-1, w-1} & \text { if } 0 \leq i<u<v<w .\end{cases}
$$

One can check that in this way we get a $K(A, 3)$ simplicial group.

\section{Eilenberg-MacLane simplicial groups $K(A, n)$}

The above two examples suggest a description for all Eilenberg-MacLane simplicial groups $K(A, n)$. This time, to color the n-skeleton of the q-simplex $\Delta_{q}$, it is enough to color all $\mathrm{n}$-simplices of the form $\left[u_{1}, u_{2}, \ldots, u_{n}, u_{n}+1\right]$, where $0 \leq u_{1}<$ $u_{2}<\cdots<u_{n} \leq q-1$.

We define

$$
K(n)_{q}=A^{\left(\begin{array}{c}
q \\
n
\end{array}\right)} .
$$

The elements of $A^{\left(\begin{array}{c}q \\ n\end{array}\right)}$ are $\left(\begin{array}{c}q \\ n\end{array}\right)$-tuples $\left(a_{u_{1}, \ldots, u_{n}}\right)_{\left(0 \leq u_{1}<\cdots<u_{n} \leq q-1\right)}$ with the index in the lexicographic order

$$
\left(a_{0,1, \ldots, n-2, n-1}, a_{0,1, \ldots, n-2, n}, \ldots, a_{q-n, \ldots q-2, q-1}\right) .
$$

For every $0 \leq i \leq q$ define

$$
\partial_{i}: K(n)_{q}=A^{\left(\begin{array}{c}
q \\
n
\end{array}\right)} \rightarrow K(n)_{q-1}=A^{\left(\begin{array}{c}
q-1 \\
n
\end{array}\right)},
$$

with $\partial_{i}\left(\left(a_{u_{1}, \ldots, u_{n}}\right)_{\left(0 \leq u_{1}<\cdots<u_{n} \leq q-1\right)}\right)=\left(b_{u_{1}, \ldots, u_{n}}\right)_{\left(0 \leq u_{1}<\cdots<u_{n} \leq q-2\right)}$, where

$$
b_{u_{1}, \ldots, u_{n}}= \begin{cases}a_{u_{1}, \ldots, u_{n}} a_{u_{1}, \ldots, u_{n-1}, i}^{(-1)^{0}} a_{u_{1}, \ldots, u_{n-2}, u_{n}, i}^{(-1)^{1}} \ldots a_{u_{2}, \ldots, u_{n}, i}^{(-1)^{n-1}} & \text { if } u_{n}=i-1 \\ a_{d^{i}\left(u_{1}\right), \ldots, d^{i}\left(u_{n}\right)} & \text { if } u_{n} \neq i-1\end{cases}
$$


The degeneracy maps are

$$
s_{i}: K(n)_{q}=A^{\left(\begin{array}{c}
q \\
n
\end{array}\right)} \rightarrow K(n)_{q+1}=A^{\left(\begin{array}{c}
q+1 \\
n
\end{array}\right)},
$$

with $s_{i}\left(\left(a_{u_{1}, \ldots, u_{n}}\right)_{\left(0 \leq u_{1}<\cdots<u_{n} \leq q-1\right)}\right)=\left(c_{u_{1}, \ldots, u_{n}}\right)_{\left(0 \leq u_{1}<\cdots<u_{n} \leq q\right)}$, where

$$
c_{u_{1}, \ldots, u_{n}}= \begin{cases}1 & \text { if } u_{n}=i, \\ a_{s^{i}\left(u_{1}\right), \ldots, s^{i}\left(u_{n}\right)} & \text { if } u_{n} \neq i,\end{cases}
$$

with the convention that if two consecutive indices $s^{i}\left(u_{j}\right), s^{i}\left(u_{j+1}\right)$ are equal then the corresponding element is trivial (that is, $a_{s^{i}\left(u_{1}\right), \ldots, s^{i}\left(u_{n}\right)}=1$ ).

REMARK 4.1. To better understand the definition of $\partial_{i}$, we should remember that the elements from $K(n)_{q}=A^{\left(\begin{array}{c}q \\ n\end{array}\right)}$ are indexed by the n-simplices $\left[u_{1}, u_{2}, \ldots, u_{n}, u_{n}+1\right]$ from $\Delta_{q}$. Also $\partial_{i}$ corresponds to $d^{i}: \overline{q-1} \rightarrow \bar{q}$. This means that the color of $\left[u_{1}, u_{2}, \ldots, u_{n}, u_{n}+1\right]$ in $\Delta_{q-1}$ is the color of $\left[d^{i}\left(u_{1}\right), d^{i}\left(u_{2}\right), \ldots, d^{i}\left(u_{n}\right), d^{i}\left(u_{n}+1\right)\right]$ from $\Delta_{q}$. If $u_{n} \neq i-1$ then $d^{i}\left(u_{n}+1\right)=d^{i}\left(u_{n}\right)+1$ and so the color of $\left[u_{1}, u_{2}, \ldots\right.$,

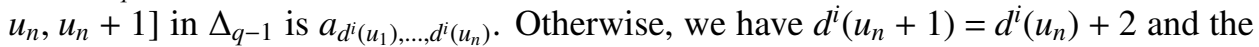
color is determined by 'homotopy', as described in the previous section for the case $n=2$. A similar argument can be made for the definition of $s_{i}$.

Theorem 4.2. $\left(K(n), s_{i}, \partial_{i}\right)$ is a $K(A, n)$ simplicial group.

Proof. We start by checking that $\left(K(n), s_{i}, \partial_{i}\right)$ is a simplicial group. Since this is a long but straightforward computation we give the details for the first step and then list the relevant information that is used in the others steps.

Step 1. $\left(\partial_{i} \partial_{j}=\partial_{j-1} \partial_{i}\right.$ if $\left.i<j\right)$. Take $\bar{a}=\left(a_{u_{1}, \ldots, u_{n}}\right)_{\left(0 \leq u_{1}<\cdots<u_{n} \leq q-1\right)} \in K(n)_{q}$. In order to prove that $\partial_{i} \partial_{j}(\bar{a})=\partial_{j-1} \partial_{i}(\bar{a}) \in K(n)_{q-2}$ it is enough to check the we have equality on each component $\left(u_{1}, \ldots, u_{n}\right)$ where $0 \leq u_{1}<\cdots<u_{n} \leq q-3$.

First notice that: (a) $u_{n}=i-1 \neq j-2$ if and only if $u_{n} \neq j-2$ and $d^{j-1}\left(u_{n}\right)=i-1$; (b) $u_{n}=j-2 \neq i-1$ if and only if $u_{n} \neq i-1$ and $d^{i}\left(u_{n}\right)=j-1$.

Case I. $u_{n} \neq i-1$ and $u_{n} \neq j-2$. The $\left(u_{1}, \ldots, u_{n}\right)$ components of $\partial_{i} \partial_{j}(\bar{a})$ and $\partial_{j-1} \partial_{i}(\bar{a})$ are equal to

respectively

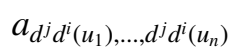

$$
a_{d^{i} d^{j-1}\left(u_{1}\right), \ldots, d^{i} d^{j-1}\left(u_{n}\right)} \text {. }
$$

Then the equality follows from the identity $d^{j} d^{i}=d^{i} d^{j-1}$ for all $i<j$.

Case II. $u_{n}=j-2 \neq i-1$ (or equivalently $u_{n} \neq i-1$ and $d^{i}\left(u_{n}\right)=j-1$ ). The $\left(u_{1}, \ldots, u_{n}\right)$ components of $\partial_{i} \partial_{j}(\bar{a})$ and $\partial_{j-1} \partial_{i}(\bar{a})$ are equal to

$$
a_{d^{i}\left(u_{1}\right), \ldots, d^{i}\left(u_{n}\right)} a_{d^{i}\left(u_{1}\right), \ldots, d^{i}\left(u_{n-1}\right), j}^{(-1)^{0}} a_{d^{i}\left(u_{1}\right), \ldots, d^{i}\left(u_{n-2}\right), d^{i}\left(u_{n}\right), j}^{(-1)^{1}} \cdots a_{d^{i}\left(u_{2}\right), \ldots, d^{i}\left(u_{n}\right), j}^{(-1)}
$$

respectively

$$
\begin{gathered}
a_{d^{i}\left(u_{1}\right), \ldots, d^{i}\left(u_{n}\right)} a_{d^{i}\left(u_{1}\right), \ldots, d^{i}\left(u_{n-1}\right), d^{i}(j-1)}^{(-1)} \\
a_{d^{i}\left(u_{1}\right), \ldots, d^{i}\left(u_{n-2}\right), d^{i}\left(u_{n}\right), d^{i}(j-1)}^{(-1)} \cdots a_{d^{i}\left(u_{2}\right), \ldots, d^{i}\left(u_{n}\right), d^{i}(j-1)}^{(-1)^{n-1}}
\end{gathered}
$$

Then the equality follows since $d^{i}(j-1)=j$. 
Case III. $u_{n}=i-1 \neq j-2$ (or equivalently $u_{n} \neq j-2$ and $d^{j-1}\left(u_{n}\right)=i-1$ ). The $\left(u_{1}, \ldots, u_{n}\right)$ components of $\partial_{i} \partial_{j}(\bar{a})$ and $\partial_{j-1} \partial_{i}(\bar{a})$ are equal to

$$
\begin{gathered}
a_{d^{j}\left(u_{1}\right), \ldots, d^{j}\left(u_{n}\right)} a_{d^{j}\left(u_{1}\right), \ldots, d^{j}\left(u_{n-1}\right), d^{j}(i)}^{(-1)} \\
a_{d^{j}\left(u_{1}\right), \ldots, d^{j}\left(u_{n-2}\right), d^{j}\left(u_{n}\right), d^{j}(i)}^{(-1)} \cdots a_{d^{j}\left(u_{2}\right), \ldots, d^{j}\left(u_{n}\right), d^{j}(i)}^{(-1)^{n-1}}
\end{gathered}
$$

respectively

$$
\begin{gathered}
a_{d^{j-1}\left(u_{1}\right), \ldots, d^{j-1}\left(u_{n}\right)} a_{d^{j-1}\left(u_{1}\right), \ldots, d^{j-1}\left(u_{n-1}\right), i}^{(-1)^{0}} \\
a_{d^{j-1}\left(u_{1}\right), \ldots, d^{j-1}\left(u_{n-2}\right), d^{j-1}\left(u_{n}\right), i}^{(-1} \cdots a_{d^{j-1}\left(u_{2}\right), \ldots, d^{j-1}\left(u_{n}\right), i^{n}}^{(-1}
\end{gathered}
$$

Then the equality follows since $d^{j}(i)=i$ and if $u_{s} \leq i$ then $d^{j}\left(u_{k}\right)=d^{j-1}\left(u_{k}\right)$.

Case IV. $u_{n}=i-1$ and $u_{n}=j-2$. The $\left(u_{1}, \ldots, u_{n}\right)$ component of $\partial_{i} \partial_{j}(\bar{a})$ is equal to

$$
\begin{gathered}
a_{u_{1}, \ldots, u_{n}}\left(a_{u_{1}, \ldots u_{n-1}, i} a_{u_{1}, \ldots, u_{n-1}, i+1}^{(-1) 0^{0}} a_{u_{1}, \ldots, u_{n-2}, i, i+1}^{(-1)^{1}} \cdots a_{u_{2}, \ldots, u_{n-1}, i, i+1}^{(-1)}\right)^{n-2} \\
\left(a_{u_{1}, \ldots u_{n-2}, u_{n}, i} a_{u_{1}, \ldots, u_{n-2}, u_{n}, i+1}^{(-1)^{0}} a_{u_{1}, \ldots, u_{n-2}, i, i+1}^{(-1)^{1}} \cdots a_{u_{2}, \ldots, u_{n-2}, u_{n}, i, i+1}^{(-1)^{n-1}}\right)^{(-1)^{1}} \cdots \\
\left(a_{u_{2}, \ldots u_{n-1}, u_{n}, i} a_{u_{2}, \ldots, u_{n}, i+1}^{(-1)^{0}} a_{u_{1}, \ldots, u_{n-1}, i, i+1}^{(-1)^{1}} \cdots a_{u_{3}, \ldots, u_{n}, i, i+1}^{(-1)^{n-2}}\right)^{(-1)^{n-1}} .
\end{gathered}
$$

The $\left(u_{1}, \ldots, u_{n}\right)$ component of $\partial_{j-1} \partial_{i}(\bar{a})$ is equal to

$$
\begin{aligned}
& a_{u_{1}, \ldots, u_{n}}\left(a_{u_{1}, \ldots u_{n-1}, i} a_{u_{1}, \ldots, u_{n-1}, i}^{(-1)^{0}} a_{u_{1}, \ldots, u_{n-2}, u_{n}, i}^{(-1)^{1}} \cdots a_{u_{2}, \ldots, u_{n}, i}^{(-1)^{n-2}}\right) \\
& \left(a_{u_{1}, \ldots u_{n-1}, j} a_{u_{1}, \ldots, u_{n-2}, u_{n}, j}^{(-1)^{0}} a_{u_{1}, \ldots, u_{n-1}, u_{n}, j}^{(-1)^{1}} \cdots a_{u_{2}, \ldots, u_{n-1}, u_{n}, j}^{(-1)^{n-1}}\right) .
\end{aligned}
$$

In the first expression all the terms with index that end in $(\ldots, i, i+1)$ appear twice with opposite sign and so they cancel each other. Since $j=i+1$, the rest of the terms are exactly those from the second expression. And so we get the equality we want.

Step 2. $\left(s_{i} s_{j}=s_{j+1} s_{i}\right.$ if $\left.i \leq j\right)$. It is enough to notice that if $i \leq j$ then the following are true: (a) $u_{n}=i$ if and only if $s^{j+1}\left(u_{n}\right)=i$; (b) if $u_{n} \neq i$ then $u_{n}=j+1$ if and only if $s^{i}\left(u_{n}\right)=j$; (c) $s^{i} s^{j+1}=s^{j} s^{i}$.

Step 3. $\left(\partial_{i} s_{j}=s_{j-1} \partial_{i}\right.$ if $\left.i<j\right)$. We use the following: (a) $u_{n}=i-1$ if and only if $s^{j-1}\left(u_{n}\right)=i-1$; (b) $u_{n}=j-1$ if and only if $d^{i}\left(u_{n}\right)=j$; (c) $d^{i} s^{j-1}=s^{j} d^{i}$.

Step 4. $\left(\partial_{j} s_{j}=\mathrm{id}\right)$. We use the following: (a) $d^{j}\left(u_{n}\right) \neq j$; (b) $s^{j} d^{j}=\mathrm{id}$

Step 5. $\left(\partial_{j+1} s_{j}=\mathrm{id}\right)$. We use the following: (a) $s^{j}(j)=s^{j}(j+1)=j$; (b) $d^{j+1}\left(u_{n}\right) \neq j$ if and only if $u_{n} \neq j$; (c) $s^{j} d^{j+1}=\mathrm{id}$.

Step 6. $\left(\partial_{i} s_{j}=s_{j} \partial_{i-1}\right.$ if $\left.i>j+1\right)$. We use the following: (a) $d^{i}\left(u_{n}\right)=j$ if and only if $u_{n}=j$; (b) if $u_{n} \neq j$ then $s^{j}\left(u_{n}\right)=i-2$ if and only if $u_{n}=i-1$; (c) $s^{j} d^{i}=d^{i-1} s^{j}$.

Finally, let us see that $K(n)$ is indeed a $K(A, n)$ simplicial group. One can notice that $\overline{K(n)}_{q}=1$ if $q<n$ and $\overline{K(n)}_{n}=A$. Next we want to show that $\overline{K(n)}_{q}=1$ if $q>n$. 
Take $\bar{a}=\left(a_{u_{1}, \ldots, u_{n}}\right)_{\left(0 \leq u_{1}<\cdots<u_{n} \leq q-1\right)} \in \overline{K(n)_{q}}$. Since $\partial_{0}(\bar{a})=1$ we get that $a_{u_{1}, \ldots, u_{n}}=1 \in A$ for all $1 \leq u_{1}<u_{2}<\cdots<u_{n} \leq q-1$. Since $\partial_{1}(\bar{a})=1$ we get that $a_{u_{1}, \ldots, u_{n}}=1 \in A$ for all $u_{1}=0<2 \leq u_{2}<u_{3}<\cdots<u_{n} \leq q-1$, then from $\partial_{2}(\bar{a})=1$ we get that $a_{u_{1}, \ldots, u_{n}}=$ $1 \in A$ for all $u_{1}=0<u_{2}=1<3 \leq u_{3}<\cdots<u_{n} \leq q-1$, and so on. Since $q>n$ we have $\partial_{n-1}(\bar{a})=1$ which gives $a_{u_{1}, \ldots, u_{n}}=1 \in A$ for all $u_{1}=0<u_{2}=1<\cdots<u_{n}=n-1$. This means that $a_{u_{1}, \ldots u_{n}}=1$ for all $0 \leq u_{1}<u_{2}<\cdots<u_{n} \leq q-1$ and so $\overline{K(n)}_{q}=1$ (the trivial group).

In particular, we have $\pi_{n}(K(n))=A$ and $\pi_{i}(K(n))=1$ for $i \neq n$ which completes our proof.

REMARK 4.3. When $n=1$ we get the classical construction of $K(A, 1)$. When $n=2$ or $n=3$ we obtain the explicit description given in the previous section.

Remark 4.4. There is an obvious connection between the simplicial group $K(G, 1)$ and the group cohomology $H^{n}(G, A)$. More precisely, $H^{n}(G, A)$ is the homology of the complex obtained by applying the functor $\operatorname{Map}(, A)$ to the complex associated to $K(G, 1)$ (here $\operatorname{Map}\left(G^{n}, A\right)$ is the set of functions from $G^{n}$ to $A$ with the group structure induced by the multiplication in $A$ ). The same statement is true for the simplicial group $K(A, 2)$ and the secondary cohomology ${ }_{2} H^{n}(A, B)$. Similarly, we can define the ternary cohomology ${ }_{3} H^{n}(B, C)$. Then for a topological space with $G=\pi_{1}(X)=1, A=\pi_{2}(X)=1, B=\pi_{3}(X)$ and $C=\pi_{4}(X)$ one can construct a homotopy invariant ${ }_{3} \kappa^{5} \in{ }_{3} H^{5}\left(\pi_{3}(X), \pi_{4}(X)\right)$.

For the general case (that is, $G=\pi_{1}(X)$ and $A=\pi_{2}(X)$ nontrivial) we have to start with a 3 -cocycle $\kappa \in H^{3}(G, A)$, take a $\kappa$-twisted product of $K(G, 1)$ and $K(A, 2)$ and obtain a complex $K\left(G, A, \kappa^{3}\right)$. Then the secondary cohomology ${ }_{2} H^{n}(G, A, \kappa ; B)$ introduced in [8] is the homology of the complex $\operatorname{Map}(K(G, A, \kappa), B)$. In the next step, start with a 4-cocycle $\lambda \in{ }_{2} H^{n}(G, A, \kappa ; B)$, take a $\lambda$-twisted product between $K\left(G, A, \kappa^{3}\right)$ and $K(B, 3)$ to obtain a complex $K(G, A, \kappa, B, \lambda)$. Then the ternary cohomology will be the homology of the complex $\operatorname{Map}(K(G, A, \kappa, B, \lambda), C)$. One is then able to associate to a space $X$ an invariant ${ }_{3} K^{5}$ in the ternary cohomology group, and so on. This is very similar with the idea used in [7] to classify simplicial groups. The main novelty is that this gives an explicit way to associate to a topological space $X$ an invariant in the appropriate cohomology theory.

\section{Secondary homology for commutative Hopf algebras}

In this section, $H$ is a commutative Hopf algebra. We want to associate to $H$ a cyclic object ${ }_{2} K(H)$. If $H$ is the group algebra $k[A]$ associated to a commutative group $A$, then ${ }_{2} K(H)$ is the linearization of the simplicial group $K(A, 2)$ described above.

Define ${ }_{2} K(H)_{q}=H^{\otimes q(q-1) / 2}$. An element of ${ }_{2} K(H)_{q}$ is a tensor:

$$
\left(\otimes h_{u, v}\right)=h_{0,1} \otimes\left(h_{0,2} \otimes h_{1,2}\right) \otimes\left(h_{0,3} \otimes \cdots \otimes h_{2,3}\right) \otimes \cdots \otimes\left(h_{0, q-1} \otimes \cdots \otimes h_{q-2, q-1}\right) .
$$


We define the maps $\partial_{i}: K_{q} \rightarrow K_{q-1}$ for all $0 \leq i \leq q$ as

$$
\begin{array}{r}
\partial_{0}\left(\left(\otimes h_{u, v}\right)_{0 \leq u<v \leq q-1}\right)=\varepsilon\left(h_{0,1} \cdots h_{0, q-1}\right) h_{1,2} \otimes\left(h_{1,3} \otimes h_{2,3}\right) \\
\otimes\left(h_{1,4} \otimes h_{2,4} \otimes h_{3,4}\right) \otimes \cdots \otimes\left(h_{1, q-1} \otimes h_{2, q-1} \otimes \cdots \otimes h_{q-2, q-1}\right), \\
\partial_{1}\left(\left(\otimes h_{u, v}\right)_{0 \leq u<v \leq q-1}\right)=\varepsilon\left(h_{1,2} \cdots h_{1, q-1}\right) \varepsilon\left(h_{0,1}\right) h_{0,2} \otimes\left(h_{0,3} \otimes h_{2,3}\right) \\
\otimes\left(h_{0,4} \otimes h_{2,4} \otimes h_{3,4}\right) \otimes \cdots \otimes\left(h_{0, q-1} \otimes h_{2, q-1} \otimes \cdots \otimes h_{q-2, q-1}\right),
\end{array}
$$

and for $2 \leq k \leq q-1$ we define

$$
\begin{aligned}
& \partial_{k}\left(\left(\otimes h_{u, v}\right)_{0 \leq u<v \leq q-1}\right)=\varepsilon\left(h_{k, k+1} h_{k, k+2} \cdots h_{k, q-1}\right) h_{0,1} \otimes\left(h_{0,2} \otimes h_{1,2}\right) \cdots \\
& \quad \otimes\left(h_{0, k-1} h_{0, k} S\left(h_{k-1, k}^{\langle 1\rangle}\right) \otimes \cdots \otimes h_{k-2, k-1} h_{k-2, k} S\left(h_{k-1, k}^{\langle k-1\rangle}\right)\right) \otimes \cdots \\
& \quad \otimes\left(h_{0, q-1} \otimes h_{1, q-1} \otimes \cdots \otimes h_{k-1, q-1} \otimes h_{k+1, q-1} \otimes \cdots \otimes h_{q-2, q-1}\right)
\end{aligned}
$$

and

$$
\begin{gathered}
\partial_{q}\left(\left(\otimes h_{u, v}\right)_{0 \leq u<v \leq q-1}\right)=\varepsilon\left(h_{0, q-1} h_{1, q-1} \cdots h_{q-2, q-1}\right) h_{0,1} \otimes\left(h_{0,2} \otimes h_{1,2}\right) \\
\otimes\left(h_{0,3} \otimes h_{1,3} \otimes h_{2,3}\right) \otimes \cdots \otimes\left(h_{0, q-2} \otimes h_{1, q-2} \otimes \cdots \otimes h_{q-3, q-2}\right) .
\end{gathered}
$$

Next we define $s_{i}: K_{q} \rightarrow K_{q+1}$ for all $0 \leq i \leq q$, by

$$
\begin{aligned}
& s_{0}\left(\left(\otimes h_{u, v}\right)_{0 \leq u<v \leq q-1}\right)=1 \otimes\left(h_{0,1}^{\langle 1\rangle} \otimes h_{0,1}^{\langle 2\rangle}\right) \\
& \quad \otimes\left(h_{0,2}^{\langle 1\rangle} \otimes h_{0,2}^{\langle 2\rangle} \otimes h_{1,2}\right) \otimes \cdots \otimes\left(h_{0, q-1}^{\langle 1\rangle} \otimes h_{0, q-1}^{\langle 2\rangle} \otimes h_{1, q-1} \otimes \cdots \otimes h_{q-2, q-1}\right), \\
& s_{1}\left(\left(\otimes h_{u, v}\right)_{0 \leq u<v \leq q-1}\right)=1 \otimes\left(h_{0,1} \otimes 1\right) \\
& \quad \otimes\left(h_{0,2} \otimes h_{1,2}^{\langle 1\rangle} \otimes h_{1,2}^{\langle 2\rangle}\right) \otimes \cdots \otimes\left(h_{0, q-1} \otimes h_{1, q-1}^{\langle 1\rangle} \otimes h_{1, q-1}^{\langle 2\rangle} \otimes \cdots \otimes h_{q-2, q-1}\right),
\end{aligned}
$$

and for $2 \leq k \leq q-1$

$$
\begin{aligned}
& s_{k}\left(\left(\otimes h_{u, v}\right)_{0 \leq u<v \leq q-1}\right)=h_{0,1} \otimes\left(h_{0,2} \otimes h_{1,2}\right) \otimes \cdots \\
& \quad \otimes\left(h_{0, k-1} \otimes h_{1, k-1} \otimes \cdots \otimes h_{k-2, k-1}\right) \otimes(1 \otimes \cdots \otimes 1) \\
& \quad \otimes\left(h_{0, k} \otimes \cdots \otimes h_{k-1, k} \otimes 1\right) \otimes\left(h_{0, k+1} \otimes \cdots \otimes h_{k-1, k+1} \otimes h_{k, k+1}^{\langle 1\rangle} \otimes h_{k, k+1}^{\langle 2\rangle}\right) \\
& \quad \otimes \cdots \otimes\left(h_{0, q-1} \otimes \cdots \otimes h_{k-1, q-1} \otimes h_{k, q-1}^{\langle 1\rangle} \otimes h_{k, q-1}^{\langle 2\rangle} \otimes \cdots \otimes h_{q-2, q-1}\right)
\end{aligned}
$$

and

$$
\begin{aligned}
& s_{q}\left(\left(\otimes h_{u, v}\right)_{0 \leq u<v \leq q-1}\right)=h_{0,1} \otimes\left(h_{0,2} \otimes h_{1,2}\right) \\
& \quad \otimes \cdots \otimes\left(h_{0, q-1} \otimes h_{1, q-1} \otimes \cdots \otimes h_{q-2, q-1}\right) \otimes(1 \otimes \cdots \otimes 1) .
\end{aligned}
$$

Finally, the cyclic action is given by $\tau_{q}: H^{\otimes q(q-1) / 2} \rightarrow H^{\otimes q(q-1) / 2}$

$$
\begin{aligned}
& \tau_{q}\left(\left(\otimes h_{u, v}\right)_{0 \leq u<v \leq q-1}\right) \\
& \quad=\otimes\left(h_{0,1}^{\langle 1\rangle} h_{0,2}^{\langle 1\rangle} \cdots h_{0, q-2}^{\langle 1\rangle} h_{0, q-1} S\left(h_{1,2}^{\langle 1\rangle} h_{1,3}^{\langle 1\rangle} \cdots h_{1, q-1}^{\langle 1\rangle}\right)\right)
\end{aligned}
$$




$$
\begin{aligned}
& \otimes\left(h_{1,2}^{\langle 2\rangle} h_{1,3}^{\langle 2\rangle} \cdots h_{1, q-1}^{\langle 2\rangle} S\left(h_{2,3}^{\langle 1\rangle} \cdots h_{2, q-1}^{\langle 1\rangle}\right) \otimes h_{0,1}^{\langle 2\rangle}\right) \\
& \otimes\left(h_{2,3}^{\langle 2\rangle} \cdots h_{2, q-1}^{\langle 2\rangle} S\left(h_{3,4}^{\langle 1\rangle} \cdots h_{3, q-1}^{\langle 1\rangle}\right) \otimes h_{0,2}^{\langle 2\rangle} \otimes h_{1,2}^{\langle 3\rangle}\right) \\
& \otimes\left(h_{3,4}^{\langle 2\rangle} \cdots h_{3, q-1}^{\langle 2\rangle} S\left(h_{4,5}^{\langle 1\rangle} \cdots h_{4, q-1}^{\langle 1\rangle}\right) \otimes h_{0,3}^{\langle 2\rangle} \otimes h_{1,3}^{\langle 3\rangle} \otimes h_{2,3}^{\langle 3\rangle}\right) \\
& \cdots \\
& \otimes\left(h_{q-3, q-2}^{\langle 2\rangle} h_{q-3, q-1}^{\langle 2\rangle} S\left(h_{q-2, q-1}^{\langle 1\rangle}\right) \otimes h_{0, q-3}^{\langle 2\rangle} \otimes h_{1, q-3}^{\langle 3\rangle} \otimes \cdots \otimes h_{q-4, q-3}^{\langle 3\rangle}\right) \\
& \otimes\left(h_{q-2, q-1}^{\langle 2\rangle} \otimes h_{0, q-2}^{\langle 2\rangle} \otimes h_{1, q-2}^{\langle 3\rangle} \otimes \cdots \otimes h_{q-3, q-2}^{\langle 3\rangle}\right) .
\end{aligned}
$$

When $H=k[A]$ we have that $\left.{ }_{2} K(H)_{q}, \partial_{k}, s_{k}, \tau_{q}\right)$ is a linearization of the simplicial group $K(A, 2)$.

Theorem 5.1. $\left({ }_{2} K(H)_{q}, \partial_{k}, s_{k}, \tau_{q}\right)$ is a cyclic module.

Proof. The proof follows exactly the same steps as in Theorem 4.2 and uses the fact that the Hopf algebra $H$ is commutative.

REMARK 5.2. If one thinks about the cyclic module $H^{(\varepsilon, 1)}$ as the cyclic module that corresponds to the first level of a 'Postnikov tower', then the second part of that 'Postnikov tower' would be a twisted product between $H^{(\varepsilon, 1)}$ and ${ }_{2} K(L)$. The analogy here is that $H$ plays the role of $\pi_{1}$ and $L$ plays the role of $\pi_{2}$ (therefore the need for $L$ to be commutative).

REMARK 5.3. In order to generalize the results from [1,2] one first needs to associate to a commutative algebra $A$ a secondary cyclic cohomology. The main problem is to define an analog of the bar resolution. We will approach that problem in a forthcoming paper.

\section{Acknowledgement}

We thank the reviewer for his comments and suggestions which contributed to the improvement of the paper.

\section{References}

[1] A. Connes and H. Moscovici, 'Cyclic cohomology and Hopf algebras', Lett. Math. Phys. 48(1) (1999), 97-108.

[2] M. Crainic, 'Cyclic cohomology of Hopf algebras', J. Pure Appl. Algebra 166(1) (2002), 29-66.

[3] S. Eilenberg and S. MacLane, 'Determination of the second homology and cohomology groups of a space by means of homotopy invariants', Proc. Nat. Acad. Sci. USA 32 (1946), 277-280.

[4] P. G. Goerss and J. F. Jardine, Simplicial Homotopy Theory, Progress in Mathematics, 174 (Birkhäuser, Basel, 1999).

[5] M. Khalkhali and B. Rangipour, 'A new cyclic module for Hopf algebras', K-Theory 27 (2002), 111-131.

[6] J. L. Loday, Cyclic Homology, Grundlehren der Mathematischen Wissenschaften, 301 (Springer, Berlin, 1992).

[7] J. P. May, Simplicial Objects in Algebraic Topology, Chicago Lectures in Mathematics (1967). 
[8] M. D. Staic, 'Secondary cohomology and k-invariants', Bull. Belg. Math. Soc. 19(3) (2012), $561-572$.

[9] C. A. Weibel, An Introduction to Homological Algebra, Cambridge Studies in Advanced Mathematics (1995).

MIHAI D. STAIC, Department of Mathematics and Statistics,

Bowling Green State University, Bowling Green, OH 43403, USA

and

Institute of Mathematics of the Romanian Academy,

PO. BOX 1-764, RO-70700, Bucharest, Romania

e-mail: mstaic@gmail.com 